(5) Adequate and enforceable legislation must be planned and in place to eliminate any possibility of discrimination based on genetic profiling.

In acknowledging the foresight of the sponsoring agencies, the final unanimous recommendation by the fellows was that workshops embodying similar Community Genetics themes should be organized on a biennial basis, with continued emphases on laboratory-based sessions.

\section{Workshop Participants}

Faculty

P. Avner (Paris), A. Bittles (Perth), M. Black (Perth), S. Chong (Singapore), E. Edkins (Perth), S. Fucharoen (Bangkok), J. Ho (Sydney), D. Kumar (Oxford), B. Lakowski (Paris), W. Lan (Shenzhen), J.J. Liu (Singapore), E. Ma (Hong Kong), B. McDonald (Sydney),
K. McElreavey (Paris), M. Patton (London), R.M. Pitchappan (Madurai), M. Reddy (Kolkata), A. Saggar (London), M. Seielstad (Singapore), S. Sullivan (Perth), W. Wang (Beijing), C. Wise (Perth), J. Yu (Shenzhen).

\section{Fellows}

F. Celep (Trabzon), L. Chalermchai (Bangkok), Y. Dai (Shenzhen), B. Das (Assam), R. Das (Chandigarh), U. Dave (Mumbai), N. Elçogliu (Istanbul), D. Hao (Shenyang), Q. Hao (Shenzhen), R. He (Shenzhen), S. Heng (Siem Reap), F. Hu (Shenzhen), A. Kaur (Amritsar), P. Kumar (Jammu), J. Li (Shenzhen), L. Liao (Shenzhen), Q. Liu (Nanning), X. Liu (Qingdao), X. Liu (Shenzhen), D. Lu (Shenzhen), F. Lu (Nanning), A. Mirfat (Jakarta), I. Nagarathinam (Chennai), R. Puri (New Delhi), W. Qian (Shenzhen), W. Qiu (Shenzhen), S. Sharma (New Delhi), D. Song (Shenzhen), B. SunarReeder (Malayta), I. Tosun (Trabzon), J. Wan (Qingdao), L. Wang (Huhehot), Y. Wang (Shenzhen), Y. Xia (Nanjing), X. Xiao (Shenzhen), J. Xie (Shenzhen), Y. Yang (Shenzhen), H. Yun (Shenzhen), C. Zhang (Shenzhen), Y. Zhang (Shenzhen).

\title{
Erratum
}

The Journal Club article 'Cascade Testing for Autosomal Recessive Disorders' (Community Genetics 2004;7:182) was written by P. Lakeman, Amsterdam. 